\title{
CORONAL MASS EJECTIONS: INITIATION AND DETECTION
}

\author{
N. GOPALSWAMY \\ Center for Solar Physics and Space Weather, Department of Physics, The Catholic University of America, \\ Washington DC 20064, USA
}

\begin{abstract}
Coronal mass ejections (CMEs) are large-scale magnetic structures expelled from the Sun due to MHD processes involving interaction between plasma and magnetic field in closed flux regions. I provide a summary of the observational signatures and current models on CME initiation. CMEs are traditionally observed using white light coronagraphs. I also provide a summary of various signatures of CMEs detected in other wavelengths, which have helped us obtain a complete picture of the CME phenomenon in the inner heliosphere.
\end{abstract}

\section{INTRODUCTION}

Coronal Mass ejections (CMEs) were first recognized as large-scale structures expelled from the Sun in the early 1970's (Tousey, 1973 using space-based coronagraphs. Thousands of CMEs have been observed since then, and their physical properties are well known (see Hundhausen, 1999; St Cyr, 2000). CMEs are currently studied extensively, owing to their role in deciding the physical conditions in the geospace; they are also important in understanding the long-term evolution of solar magnetism since they remove significant amounts of magnetic flux along with material (see, e.g., Low, 2001). CMEs are responsible for driving interplanetary (IP) shocks (Sheeley et al., 1985), and as a consequence, the solar energetic particles (SEPs, see e.g., Tylka, 2001). How CMEs are initiated is still a mystery. In recent years, spacecraft observations at various wavelengths (apart from the traditional white light) have provided extensive information on the source regions and the initial phase of CMEs. To understand CME initiation, we need to have complete information on the structure and dynamics of the preeruption, closed magnetic field regions on the Sun. I summarize the observed signatures at various layers of the solar atmosphere corresponding to the solar source of CMEs. I also summarize new insights we have gained by combining the white light data with images at other wavelengths.

\section{INITIATION}

CMEs originate from large-scale closed magnetic field regions such as active regions and filament/prominence regions. Active regions and filament regions often form complexes. Large-scale closed filed lines can also be found interconnecting active regions (Svestka). During solar minima, the equatorial streamer belt constitutes a dominant closed field structure. Thus, we need to look at the evolution of the magnetic field for clues to the CME initiation. While we can reliably measure the photospheric magnetic field, it is difficult to do so in the outer layers; we can infer the topology of the field lines from coronal images. Thus, we need to look for signatures of spatio-temporal changes in the structure of closed field regions in order to understand CME initiation.

\subsection{PRE-ERUPTION STRUCTURES}

What kind of closed field regions will result in eruption? The helmet-streamer structure, known for a long time from eclipse pictures, is a large-scale closed field region. The closed field part of the streamer deforms to become the frontal structure of the CME, followed by the coronal cavity and the prominence core (Hundhausen, 1999). Thus the " three-part structure" of CMEs (see Fig. 1) can be readily observed in the pre-eruption phase, and is now commonly observed by inner coronal imagers such as the Yohkoh mission's Soft X-ray Telescope (SXT) and the Solar and Heliospheric Observatory's (SOHO) Extreme-ultraviolet Imaging Telescope (EIT). The preeruption configuration in active regions is probably similar, except for the height of the filament and the strength of the overlying magnetic field. Transequatorial and interconnecting structures may result in CMEs without a prominence core. However, multi-arcade eruptions (Webb et al., 1997; Gopalswamy, 1999) that span more than one closed region may still contain a prominence core from one of the underlying flux systems (Gopalswamy et al. 1999a; Gopalswamy and Thompson, 2000). 
Sheared magnetic structures, inferred from photospheric magnetograms, have been thought to be indicative of imminent eruption (Ambastha et al., 1993). Combining vector magnetograms with the Yohkoh soft X-ray images, Falconer (2001) has identified two characteristics of active regions that may be useful in discriminating CMEproductive active regions: the length of the strong-field, strong-shear main neutral line and the global net current in the active region. Above certain threshold values of these parameters, production of CMEs is likely. The coronal manifestation of the photospheric shear is the sigmoidal structure in active regions, observed as S- and reverse S-shaped features (Rust and Kumar, 1996) in soft X-ray images. Statistically, sigmoidal regions have been found to be more eruptive than the non-sigmoidal regions (Canfield et al., 1999; Glover et al., 2000). Sigmoidal structures are replaced by arcades in the post eruption phase (Sterling and Hudson, 1997). Most of the events studied by Canfield et al. (1999) correspond to a period with no white light observations, so their association with CMEs is not clear. Systematic analysis of CMEs originating from sigmoidal regions is necessary before making firm conclusions.
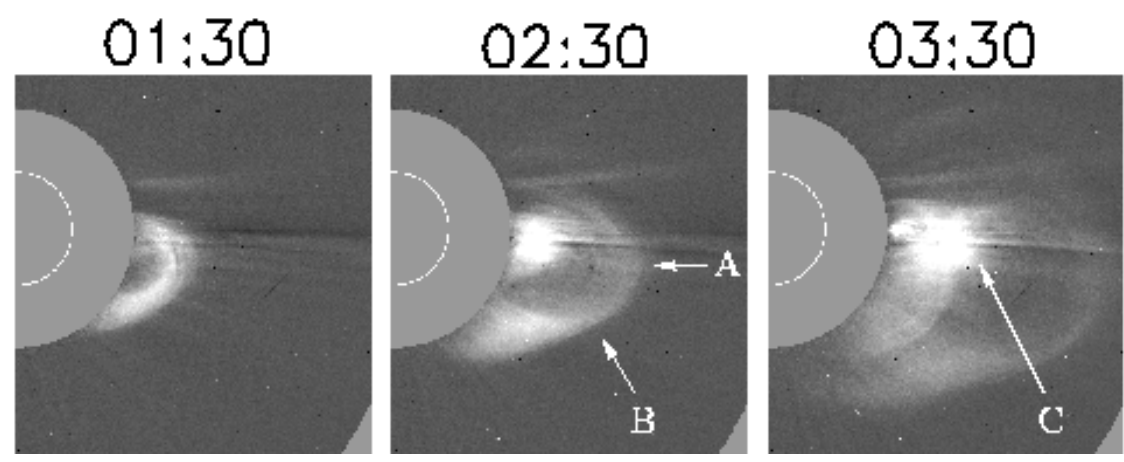

Fig. 1. Frontal structure (A, B), core $(\mathrm{C})$, with a dark void in between for the 1997 February 7 CME.

The formation and eruption of prominences is one of the central issues of CME initiation. In fact some authors consider the prominence eruption as the cause of CMEs (Filippov, 1996; Wu et al., 2000), contrary to the arguments that prominence cores are not energetically favorable to cause CMEs (Hundhausen, 1999). The complex relationship between the filament, coronal cavity and the frontal structure before and during the eruption is a subject of current debate (see e.g., Rust, 2001; Linker et al., 2001; Low, 2001). Many CMEs in the IP medium have been found to have flux rope structure (magnetic clouds), although it is not clear which part of the CME becomes the cloud (see e.g., Bothmer and Schwenn, 1994). Recently, certain concave-outward white light features in the coronagraph field of view have been interpreted as flux ropes (Chen et al. 1998). The question is whether the flux ropes are pre-eruption structures or they are formed during eruption (Low and Hundhausen, 1995; Gosling, 1990). The flux rope structure naturally provides the necessary "dip" where the filament can reside (e.g. van Ballegooijen and Martens, 1989) and a reduction in the magnetic flux could cause the flux rope to erupt (Linker et al., 2001). Shearing and twisting of magnetic field lines can produce the necessary dips to support the prominence (Antiochos et al., 1994). Another idea is that the prominence is simply a flowing material and no dip is needed for support (Martin and McAllister, 1997).

\subsection{PRE-ERUPTION EVOLUTION}

Flux emergence has been considered one of the as a possible trigger of CMEs, which initiates small-scale reconnection near the filaments. Feynman and Martin (1995) examined the magnetic flux in the source regions of CMEs associated with filament eruptions and found that they occurred after the emergence of substantial amounts of new flux in the vicinity of the filaments. One of the requirements of the eruption is a "favorable" orientation of the newly emerging flux with respect to the preexisting flux in such a way that small-scale reconnection can take place. Using numerical simulation, Chen and Shibata (2000) demonstrated the eruption of arcades overlying the filaments as CMEs due to reconnection between emerging and existing magnetic field lines. However, Wang and Sheeley (1999) studied a set of filament eruptions and concluded that flux emergence serves as a catalyst, rather than a trigger of the filament eruption. Subramanian and Dere (2001) studied a larger sample of CME events and found flux emergence in many cases, but there were eruptions with no substantial flux emergence. They also found eruptions with no substantial flux emergence. The study of Subramanian and Dere (2001) is consistent with the conclusions of Wang and Sheeley (1999).

In another study, Lara et al. (2000) found significant changes in the flux at the time of CMEs in small subregions within the overall region of eruption. The flux change was also observed during flares without CMEs, but the 
largest changes were found at the times of CMEs. When the flux from the entire region of eruption was tracked, the changes were not significant. The flux changes were also found to be substantial only close to the photospheric neutral line. The time resolution of the SOHO/MDI magnetograms used for this study was rather poor $(90 \mathrm{~min})$. Similar study with a better time resolution may be able to shed light on the importance of localized flux changes in the eruptive region.

Sometimes, a prolonged ( $\sim 1 \mathrm{hr})$ weak dimming in EUV and X-rays occurs in the eruption region, followed by a deep dimming corresponding to the CME (Gopalswamy et al., 1999a; Gopalswamy and Kaiser, 2001). Coronal dimming represents depletion of material in the corona (see e.g., Hudson et al., 1998). Therefore, pre-eruption dimming may correspond to small-scale opening of magnetic field lines in the eruption region.

\subsection{PRE-ERUPTION ENERGY RELEASE}

Both thermal and nonthermal signatures of energy release have been reported on various occasions. Thermal signatures are in the form of small-scale X-ray or EUV brightening in the eruptive region. Nonthermal signatures are in the form of radio bursts of type III, due to energetic electrons carrying tens of keV. Fig. 2 shows a compact X-ray brightening (pointed by arrow in Fig. 2) near the location of a filament that subsequently erupted (see also Gopalswamy, 1999). It took about 3 hours after the brightening for the filament to completely disappear. The filament eruption was associated with the CME of 1998 January 21. The compact brightening was located at the apex of a triangular dimming region. While the dimming was observed both in X-ray and EUV images, the compact brightening showed up only in X-ray images suggesting that the latter is indeed a hot structure.

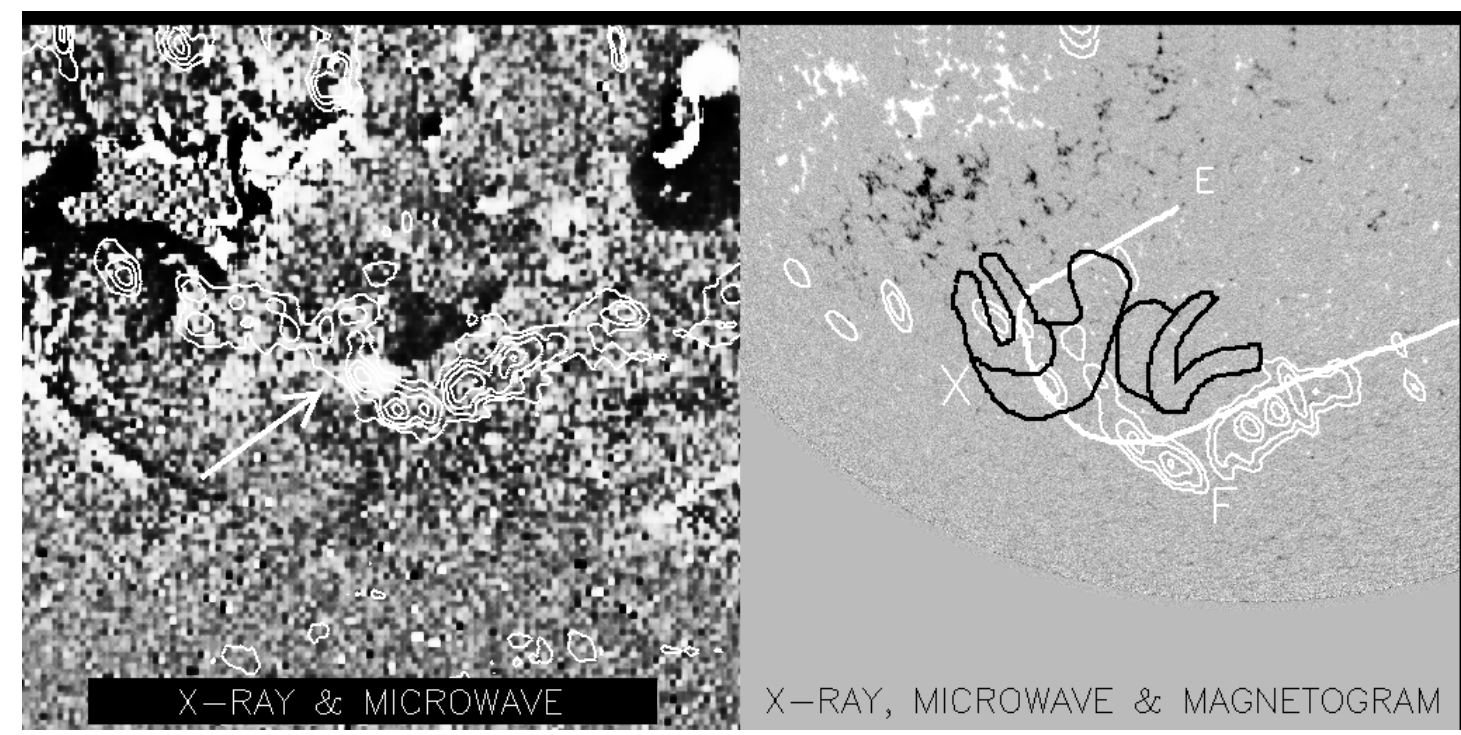

Fig.2. (left) X-ray brightening before the eruption of the filament and (right) magnetic neutral line and posteruption X-ray structures superposed on a SOHO/MDI magnetogram. The filament is plotted as white contours.

Many years ago, Jackson et al. (1978) found that, statistically, the number of type III radio bursts peaked about an hour before CMEs, suggesting that small-scale energy release in the form of energetic electrons preceded the CME eruption. Using the Clark Lake multifrequuency radioheliograph images, Gopalswamy et al. (1987) reported on precursor type III bursts located above the region of eruption. Recently, Marque et al. (2000) found nonthermal radio bursts originating near a filament that subsequently erupted.

The evidence for small-scale energy release is consistent with the flux emergence reported by Feynman and Martin (1995) and the simulation studies of Chen and Shibata (2000) and points to the possibility of reconnection in the vicinity of filaments in the pre-eruption phase. It is however, not clear whether the small-scale energy release is a consequence of the lifting off of the cavity or overlying arcade. In the scenario of multi-arcade eruption proposed by Antiochos et al. (1994), reconnection takes place in the region overlying the eruptive structures, redistributing the flux and hence enabling underlying flux system to erupt. Observational signatures of this reconnection are expected in the peripheral regions of the eruptive structure. Current ideas and models that address the structure, evolution and energy release in the pre-eruption phase are summarized in Table 1. 
Table 1. Summary of some current ideas and models

\begin{tabular}{|l|l|l|l|}
\hline Model & Pre-Eruption Structure & Pre-eruption Evolution & $\begin{array}{l}\text { Pre-eruption Energy } \\
\text { Release }\end{array}$ \\
\hline $\begin{array}{l}\text { Forbes et al., 1994, } \\
\text { Linker, et al. 2001 }\end{array}$ & $\begin{array}{l}\text { Flux rope in bipolar field: } \\
\text { either emerges or forms in } \\
\text { the corona }\end{array}$ & $\begin{array}{l}\text { Flux decrease/changes } \\
\text { leading to loss of } \\
\text { equilibrium }\end{array}$ & None \\
\hline Chen and Shibata, 2000 & $\begin{array}{l}\text { Flux rope in multipolar } \\
\text { field }\end{array}$ & $\begin{array}{l}\text { Flux emerges consistent } \\
\text { with reconnection }\end{array}$ & $\begin{array}{l}\text { Reconnection energization } \\
\text { at the site of emergence }\end{array}$ \\
\hline Antiochos et al., 1994 & $\begin{array}{l}\text { Sheared arcade in } \\
\text { multipolar field }\end{array}$ & $\begin{array}{l}\text { Continued shearing } \\
\text { at connection energization }\end{array}$ \\
\hline Wu et al., 2000 & $\begin{array}{l}\text { Flux rope with overlying } \\
\text { streamer in the solar wind }\end{array}$ & $\begin{array}{l}\text { Increase in the azimuthal } \\
\text { flux or shear of the } \\
\text { streamer field }\end{array}$ & None \\
\hline Chen et al., 1997 & Flux rope in equilibrium & $\begin{array}{l}\text { Increase in the azimuthal } \\
\text { flux }\end{array}$ & None \\
\hline
\end{tabular}

\section{DETECTION}

Material ejection from the Sun has been known for a long time from meterwave radio observations (moving type IV bursts, Boischot, 1957) and from H-alpha observations (Dodson and McMath, 1952). Mass ejection was also inferred from the estimated speeds of agencies responsible for metric type II bursts (Payne-Scott, 1947). The concept of mass ejections ("plasma clouds") has been in use for a long time (Morrison, 1954; Gold, 1955). How these ejections were related to CMEs as we know today became clear only in the early seventies, when the full glory of CMEs could be appreciated in white light coronagraph images (Tousey, 1973). Starting from Skylab, a number of non-coronagraphic signatures of CMEs in X-rays, EUV and microwaves were also recognized and are now used extensively to identify the early phase of frontside CMEs. IP shocks are responsible for IP type II bursts (Cane et al., 1987) and SEPs, and are driven by CMEs. CMEs in the solar wind were found in the data acquired by IMP, Helios and Voyager spacecraft (Burlaga et al. 1981) as magnetic clouds or ejecta. Interplanetary scintillation (IPS) observations are also capable of detecting CMEs over the entire Sun-Earth distance (Manoharan et al., 1995; Tokumaru et al, 1999).

\subsection{WHITE LIGHT}

CMEs are detected in white light the same way the corona is observed during eclipses: the photospheric light Thomson-scattered from the free electrons in the corona is gathered by the optical instrument. In a coronagrpah, an "artificial eclipse" is produced by an occulting disk to block the direct photospheric light. The white light observations do not depend on the temperature of the plasma electrons that scatter the photospheric light, so that all the substructures of the CME (see Fig. 1) are visible in coronagraphic images. Intensity of the Thomsonscattered light is a measure of the total electron content along the line of sight, which can be used to estimate the mass of CMEs. A white light CME is usually described in terms of its sky plane speed, full width and position angle measured from solar north. The CME speed is usually measured by tracking a feature of the CME in successive images. The measured speed is the sky plane speed, a lower limit to the true speed except for the CMEs originating from very close to the limb. In addition to coronagraphs, Jackson and Webb (1995) used the Helios photometer data to reconstruct CMEs over much larger distances, although the spatial resolution was much poorer. These authors found an increase in CME mass when compared to what was observed close to the Sun. Coronagraphs on future spacecraft such on the STEREO mission may be able to obtain true speeds over the entire inner heliosphere.

\subsubsection{Halo CMEs}

Since the coronal density decreases rapidly away from the Sun, CMEs are best observed by coronagraphs when they are ejected close to the limb. CMEs originating far from the limb are also observed, as enhancements surrounding the occulting disk and hence called "halo CMEs" (Howard et al., 1982). Although this interpretation was considered questionable (St. Cyr and Hundhausen, 1988), halo CMEs are now routinely observed, thanks to the high sensitivity of the SOHO/LASCO coronagraphs. The halo CMEs also confirmed the three-dimensional 
nature of CMEs (Howard et al., 1982). CMEs heading both toward and away from Earth appear as halos, so one needs additional information on the eruption from EUV, X-ray or $\mathrm{H}$-alpha observations to determine the direction (see, Fig. 3).

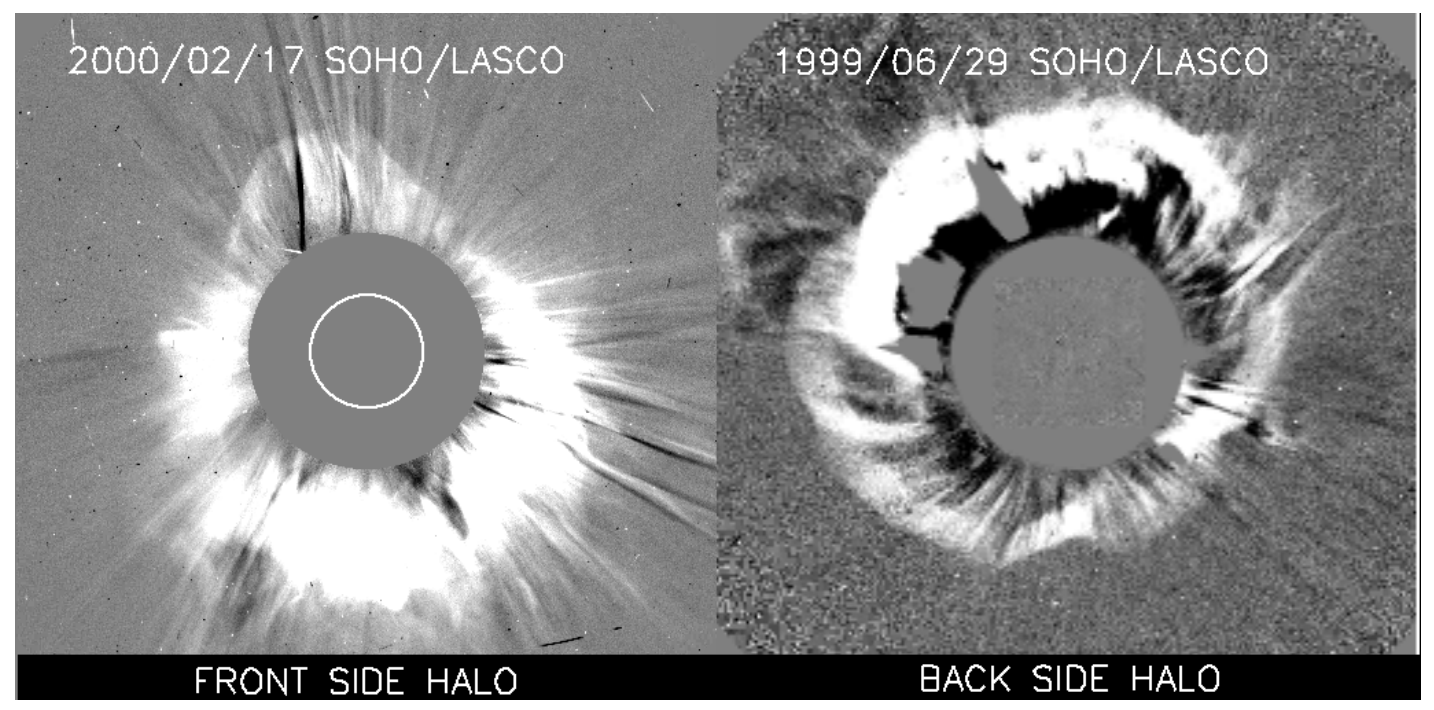

Fig. 3. Examples of front side (left) and backside (right) halos. In the right side image, an EIT difference image is superposed to shows no disk activity, because the CME originated on the backside of the Sun and is moving away from the observer.

Recently, Sheeley et al. (1999) found that for a set of fast halo CMEs, the height-time plot indicated deceleration. Since the measured heights of halo CMEs are subject to projection effects, the true heights of halo CMEs when they are at the edge of the coronagraph field of view is much larger $\left(>>30 R_{s}\right)$ than observed. CMEs are expected to decelerate at large heights because they have speeds very close to the solar wind speed at $1 \mathrm{AU}$, irrespective of their starting speed near the Sun (Lindsay et al., 1999; Gopalswamy et al., 2000d). The subset of CMEs that produces radio emission at long wavelengths also shows predominantly deceleration even when they originate close to the limb (Gopalswamy et al., 2000b). This may be due to the fact that the drag force is significantly higher for faster events.

\subsection{X-RAYS AND EUV}

The early life of CMEs cannot be studied using white-light coronagraphs because of the occulting disks employed to block the photospheric light. Inner coronal imagers in X-rays, microwaves and EUV can detect most of the features associated with the onset of CMEs (Gopalswamy 1999; Hudson 1999; Hudson and Cliver 2001). At these wavelengths, the radiation emitted in the corona is imaged, unlike in white light. While most of the CME-related near-surface phenomena were discovered during the Skylab era, detailed studies became possible after the advent of SXT and SOHO/EIT. Primary CME related signatures at these wavelengths are coronal dimming, ejected plasmoid and arcade formation. EUV wave transients (Thompson et al., 1999) and global enhancement (Gopalswamy et al., 1999c) are the two newer signatures.

Coronal dimming essentially represents depletion of coronal material due to a CME (see e.g., Hudson and Webb, 1997). Care must be taken to make sure that the dimming is not due to temperature change in the case of spectral line imaging. Coronal dimming is best observed in the EIT 195 angstrom images and displays considerable variation from event to event. EIT images often show the dimming signature in the direct images, while one has to use difference images to identify the dimming signature in X-rays. As we pointed out before, there are weak and prolonged dimming signatures in the pre-eruption phase and intense dimming during the eruption phase. Combined EUV and white light observations suggest that the coronal dimming corresponds to the depletion of the coronal material, which later becomes the CME in the coronagraph field of view (Gopalswamy et al., 1999b; Thompson et al., 2000).

The ejected plasmoid is most likely the heated prominence core of CMEs. The eruptive prominence can be seen in absorption and in emission depending upon its temperature (Gopalswamy et al., 1999c). X-ray ejecta can also be seen on the disk in X-ray movies (Gopalswamy et al., 1997). Recently, Nitta and Akiyama (2000) studied a handful of X-ray ejecta associated with LASCO CMEs and found them to be slower than the CMEs, consistent with what is known from white light observations. The CME leading edge is not seen in X-rays because the flare 
loops are usually much brighter. Under rare circumstances when there are no hot structures, it may be possible to detect the leading edge of the CME itself (Gopalswamy et al., 1996).

Arcade formation is a post-eruption signature (see, e.g., McAllister et al, 1996) often used to identify the location and extent of an eruption. Some of the eruptions with extremely weak near-surface activities have been identified in this way (see, e.g., Webb et al., 1998). Arcade formation is also observed in EUV, but not so complete as in Xrays. Fig. 4 shows an arcade in X-rays and in EUV for the 1998 Jan 25 CME. An interesting result of this observation is that different loops of the arcade had different temperatures. Some of the darker loops in the EUV arcade were hotter, as evidenced by the fact that they were bright in X-rays.

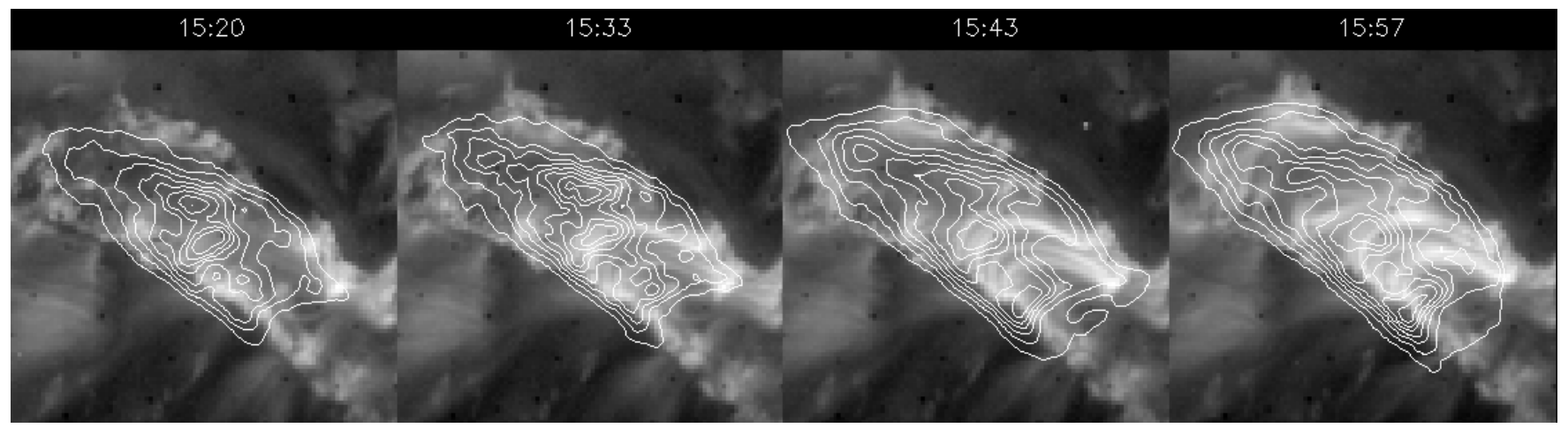

Fig. 4. Arcade formation following the 1998 January 25 CME. Contours of X-ray intensity (from Yohkoh/SXT) are plotted on EUV intensity (from SOHO/EIT).

The EIT transients are newly discovered wave-like phenomenon representing the early phase of CMEs (Thompson et al., 1999). One of the interpretations is that the EIT waves are fast mode MHD waves associated with solar eruptions and that they eventually become MHD shocks to produce metric type II bursts (Klassen et al., 2000). Under some circumstances, the EIT waves may be interpreted as shocks, especially when they have the "brow" structure (Gopalswamy, 2000) and are closely associated with H-alpha Moreton waves (Thompson et al., 2000), and metric type II bursts (Gopalswamy et al., 2000c). Khan and Hudson (2000) observed some transient features in X-rays during flares associated with transequatorial eruptions. They believe that the transients they observed are counterparts of EIT waves and may be the manifestations of coronal shocks responsible for metric type II bursts.

A relatively new X-ray signature, the "global enhancement," was identified by Gopalswamy et al. (1999d) during the filament eruption event of 1994 April 30. A weak, large-scale and diffuse enhancement was imaged by Yohkoh/SXT. The area of enhancement was much larger than the region of arcade formation. The global enhancement was interpreted as the early form of the CME itself. Later on, a number of such events were also observed in EIT 195 A images during the early phase of CMEs. A detailed investigation of this phenomenon is needed to determine whether it can be used to detect disk CMEs.

\subsection{RADIO}

In radio, both thermal and nonthermal signatures of CMEs can be detected. Thermal emission depends on the temperature, density and magnetic field of the region as well as on the observing frequency. Nonthermal emission depends on quantities such as the density and energy of the nonthermal electrons. A number of mechanisms operate in producing thermal and nonthermal radio emission, so a careful identification of the mechanism is crucial in using the radio technique for CME studies.

\section{$\underline{\text { 3.3.1 Microwaves }}$}

Filament/prominence eruptions (Hanaoka et al., 1994; Gopalswamy et al., 1996, Hanaoka and Shinkawa, 1998), arcade formations (Hanaoka, et al., 1994) and even coronal dimming can be studied using microwave images. Filament eruptions are most spectacular in microwaves and provide quantitative information on the eruptive process (Gopalswamy and Hanaoka, 1998). Comparison between eruptive prominences in H-alpha and in microwaves in Fig. 5 shows that microwave images provide a more complete picture of the eruptive prominence. 
Coronal dimming in microwaves is somewhat difficult to observe because the coronal structures are optically very thin. Nevertheless, the observed radio dimming is consistent with X-ray dimming, confirming the depletion of material from the corona (see Fig.6).
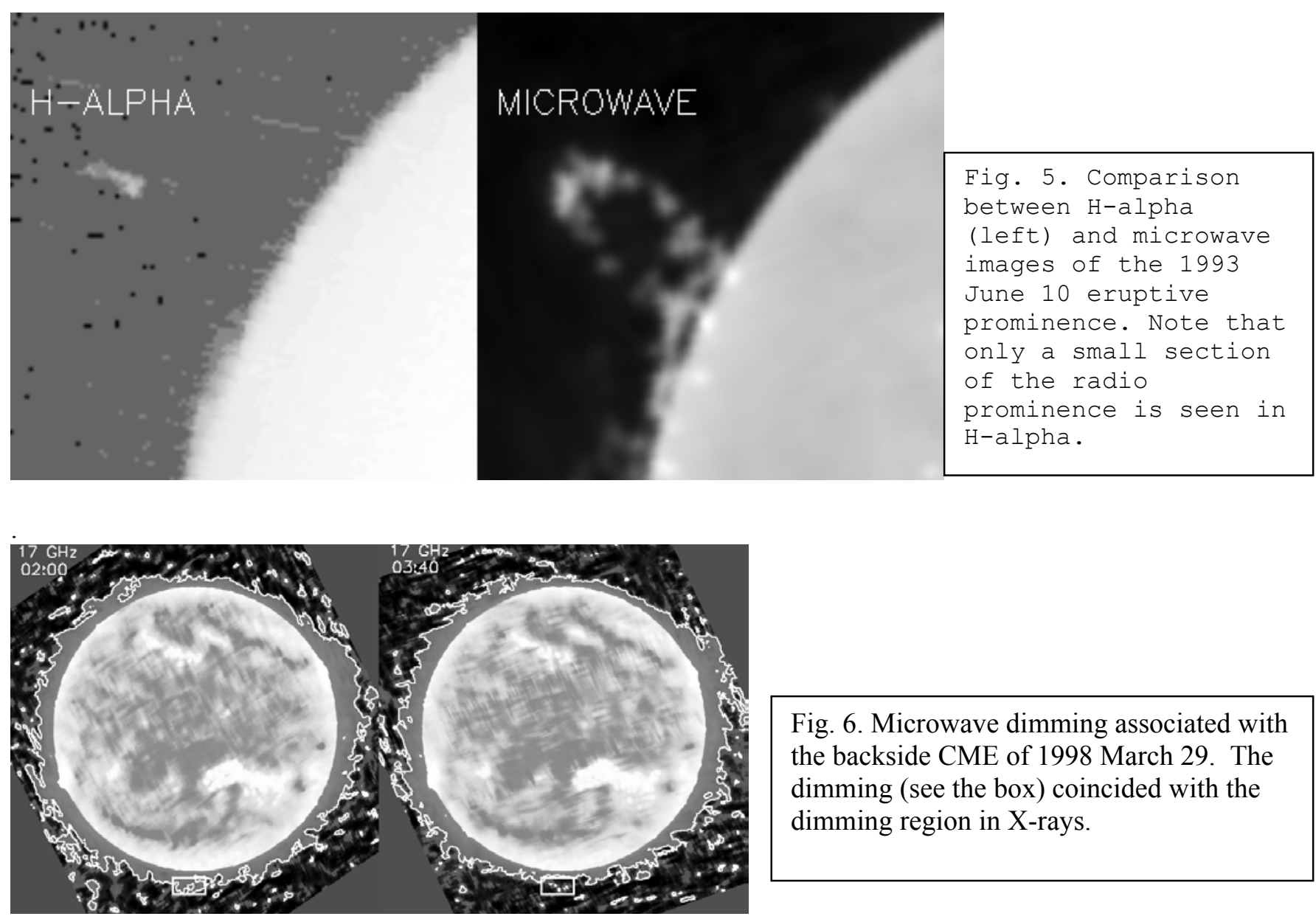

Fig. 6. Microwave dimming associated with the backside CME of 1998 March 29. The dimming (see the box) coincided with the dimming region in X-rays.

\subsubsection{Meterwaves}

In meter waves, various types of radio bursts are observed which are produced by nonthermal electrons escaping from the region of eruption along open field lines (type III bursts), electrons confined to shock front (type II bursts) or trapped in moving coronal structures (moving type IV bursts). Type II and type IV bursts are closely associated with CMEs, although it is not clear whether the shock is driven by the CME or the CME presents favorable conditions for shock propagation (Cane, 1984; Gopalswamy et al., 1998; Cliver et al., 1999).

The moving type IV bursts come in three varieties: advancing fronts, expanding arches and isolated plasmoids (see the review by Stewart, 1985). The isolated sources originate from heated prominence material, also detected in X-rays and EUV. The advancing fronts and expanding arches must be structures associated with the CME itself. Gopalswamy and Kundu (1989) tracked a moving type IV burst for several solar radii at three frequencies and found that the radio source moved roughly with the speed of the associated white light CME. They were also able to infer the structure of the loops by connecting the centroids of the radio sources. These substructures are "visible" only because of the nonthermal particles trapped in them. Recent images made by the Nancay radioheliograph show radio structures that correspond to the frontal structure of CMEs (Maia et al., 2000).

Since CMEs are denser than the ambient corona, they must be optically thick at low metric frequencies. For this reason, coronal streamers are clearly visible in meter-decameter images. Sheridan et al. (1978) detected changes in the radio streamer associated with a slow CME observed by Skylab. Gopalswamy and Kundu (1992) imaged a CME using the Clark Lake multifrequency radioheliograph at three frequencies and were able to estimate the mass of the CME from radio observations alone. The main difficulty in imaging CMEs at these frequencies is the simultaneous occurrence of nonthermal bursts that easily mask the thermal emission. 

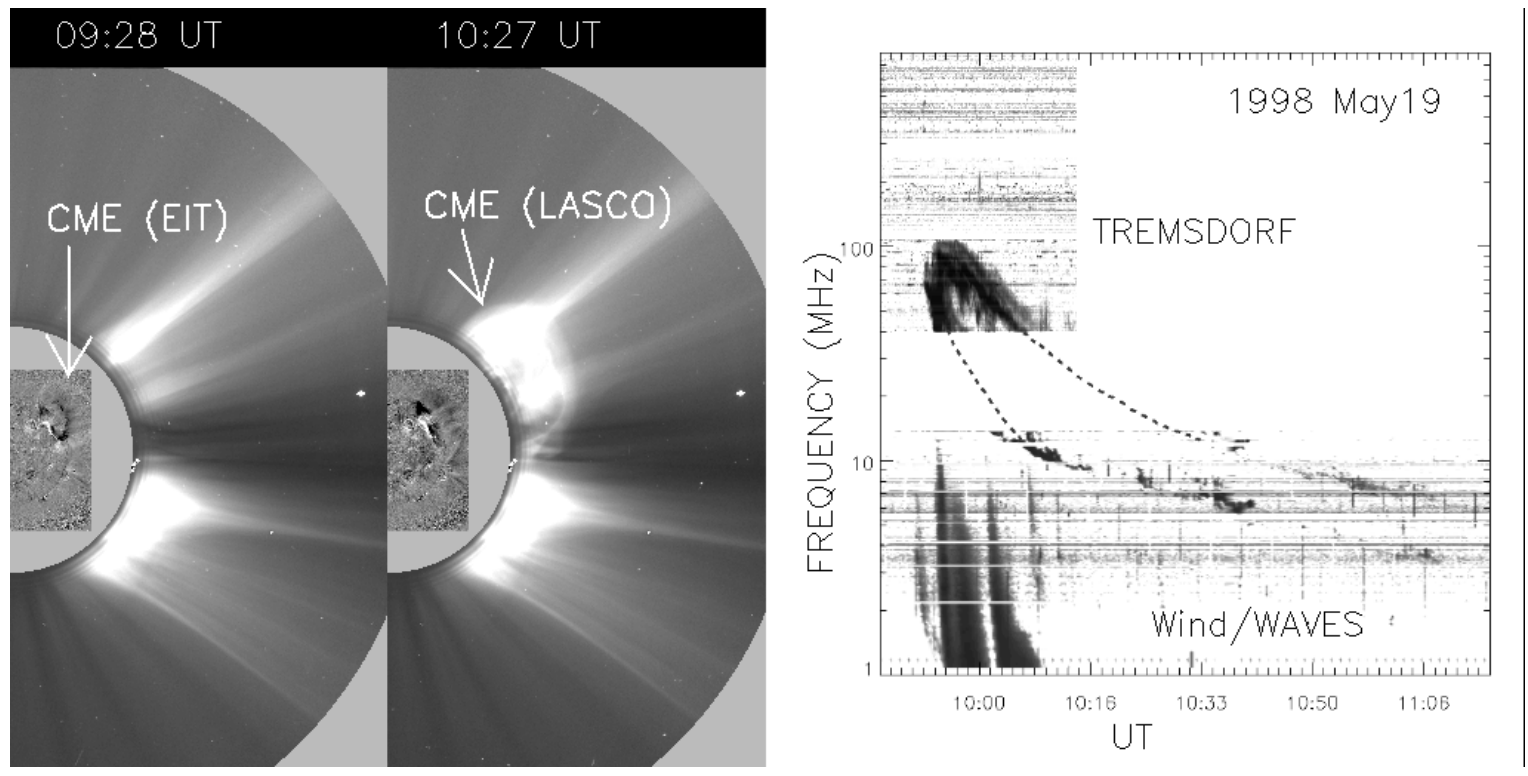

Fig. 7. Decameter-Hectometric type II burst and type III bursts due to shock-accelerated electrons (right) associated with the 1998 may 19 CME observed by SOHO/EIT and LASCO (left).

\subsubsection{Decameter-Hectometric Waves}

One of the most recent additions to instruments which detect CMEs in radio is the RAD2 receiver of the Radio and Plasma Wave (WAVES) experiment (Bougeret et al., 1995) on board the Wind spacecraft. The DecameterHectometric (DH) domain corresponds to $21 \mathrm{~m}$ to $300 \mathrm{~m}$ in wavelength (14 to $1 \mathrm{MHz}$ in frequency) and fills the gap that existed for a long time between the ground based observations $(>15 \mathrm{MHz})$ and space based observations $(<2 \mathrm{MHz}$ ). Radio bursts associated with shocks (type II), electron beams escaping from the shock front (type III bursts) and moving coronal structures (type IV bursts) are observed in this domain. Fig. 7 shows a CME from the northwest quadrant of the sun that produced a type II burst in the metric domain and continued into the DH domain. Note that there were no radio bursts when the CME was at a height of $1.5 \mathrm{R}_{\mathrm{s}}$ as seen in the EIT image at 09:28 UT. The type II burst starts only at 09:50 UT, with simultaneous type III bursts due to shock-accelerated electrons (Cane et al., 1981). Gopalswamy et al. (2000b) showed that most DH type II bursts are associated with faster and wider CMEs. DH type II bursts provide an essential tool to detect faster and wider CMEs, which are potentially more geoeffective when Earth-directed. While the relation between metric and DH type II bursts is controversial (Reiner et al., 2001), there is no doubt that the DH type II bursts are indicative of CMEs leaving the outer corona (Reiner and Kaiser, 1999; Gopalswamy et al., 2001).

\subsection{IN-SITU OBSERVATIONS}

Spacecraft instruments can directly sample the CMEs. Since CMEs propagate long distances before reaching the spacecraft, they are considerably evolved and hence it is difficult to relate the in situ observations to the CME substructures observed near the Sun. The generic term used for CMEs in the solar wind is 'ejecta'. Interplanetary CME (ICME) is another general term used to describe the CME-like disturbance in the solar wind. Most often one encounters the following sequence of structures detected by a spacecraft: IP shock, sheath, and ejecta. The ejecta can contain ordered magnetic field, in which case it is termed as a magnetic cloud (Burlaga et al., 1981). Disordered magnetic fields are also observed in the ejecta (e.g., Hundhausen, 1972) and hence magnetic clouds constitute only a subset of the IP ejecta. On rare occasions, one observes cool dense material towards the end of the ejecta that resemble the prominence resting at the bottom of the coronal cavity in the pre-eruption phase of CMEs (Burlaga et al., 1999; Gopalswamy et al., 1998). Based on this correspondence, one can make the associations between CMEs observed near the Sun and in the solar wind listed in Table 2.

\begin{tabular}{|l|l|}
\hline CME near the Sun & CME in the Solar Wind \\
\hline Coronal Shock & IP Shock \\
\hline Frontal Structure & Sheath \\
\hline Cavity & Magnetic Cloud/Ejecta \\
\hline Prominence Core & Pressure Plug \\
\hline
\end{tabular}

Table 2. Correspondence between the substructures of CMEs and ICMEs. 
The fact that the prominence material is rarely observed in the solar wind is consistent with the fact that the prominence core of CMEs heat up early on as they are visible in X-rays and hence may be indistinguishable from other coronal structures.

Identification of CME signatures in the solar wind is complicated and is often not unique (see e.g., Neugebauer, 1997 and references therein). Commonly used signatures are: counterstreaming superthermal electrons and protons, helium abundance enhancements, anomalously low electron and proton temperatures, strong magnetic fields, smooth rotation of magnetic fields, low plasma low magnetic field variance, flux reduction of low energy cosmic rays, and unusual ionization states and elemental abundances. It is important to point out that not all signatures are seen in all events and, different signatures do not coincide temporally and spatially.

\subsection{INTERPLANETARY SCINTILLATIONS (IPS)}

By observing a large number of natural radio sources distributed all around the Sun, it is possible to produce allsky maps to detect IP disturbances (see, e.g., Manoharan et al., 1995; Tokumaru et al., 2000). Tokumaru et al. (2000) studied the three-dimensional propagation of four IP disturbances between 0.2 and 1 AU, using IPS data. They were able to show that the propagation speed depends on both the latitude and longitude, and that the direction of maximum speed is not along the flare normal. The disturbances seem to be distorted by the slow and fast solar winds. Deceleration of the IP disturbances has also been detected, sometimes in steps (Manoharan et al., 2001). One of the major advantages of the IPS techniques is that it can track CMEs throughout the inner heliosphere while other techniques are limited to near-Sun or near-Earth domains. It has been shown that the scintillation index, which is a measure of the density fluctuations in the medium, is proportional to the density in the medium (Moore and Harrison, 1994). In the coronagraph field of view, a CME represents a density enhancement with respect to the ambient. The frontal structure and core are denser, while the cavity has a density less than the ambient. For fast events, there is also a shock ahead of the CME with a density jump. Thus it is not clear which part of the CME is detected by the IPS technique. A careful comparison between coronagraphic and higher resolution IPS observations of more CMEs is needed to settle this issue. Future observations of CMEs over the entire heliosphere by the STEREO mission will provide a good opportunity to cross-calibrate between IPS and white light observations of CMEs.

\section{CONCLUDING REMARKS}

We have discussed most of the observational signatures related to the initiation and detection of CMEs, mainly using imaging data at various wavelengths. It is clear that we need to put together multiwavelength, multiinstrument data to obtain a complete picture of the CME phenomenon. There are also other ways of detecting CMEs based on their ability to produce SEPs via IP shocks (see e.g., Tylka, 2001), complex compositional signatures (see e.g., Galvin, 1997), and ejecta signatures at 1 AU (Richardson, 1997). The CME phenomenon is intimately connected to solar magnetism as we can infer from signatures at the solar surface. However, we do not fully understand how the evolution of closed field regions result in CMEs. It is not clear if all the CMEs are produced the same way. If the complex interaction between plasma and magnetic field is the basic process in all CMEs, differences in CMEs may be understood in terms of the magnitude and duration of the propelling force. This approach may also clarify whether the difference between fast, constant speed, flare-related CMEs and slow, accelerating, prominence-related CMEs is just quantitative or qualitative. In order to understand the relation between CMEs near the Sun and ICMEs, one has to consider the interaction between CMEs and the background solar wind as well as their internal changes during propagation. A proper synthesis of all the observations from the solar surface to the inner heliosphere might eventually answer this question. The front-side halos (Earthdirected CMEs) are of importance to space weather because the severest of geomagnetic storms are caused by such CMEs (see e.g., Gosling et al., 1991). Considerable progress has been made in recent times, and it is now possible to study the correspondence between Earth-directed CMEs and solar wind disturbances as well as their geoeffects (Webb et al, 2000; Gopalswamy et al., 2000d). Ironically, it is most difficult to detect and measure the speeds of these CMEs. We need to develop methods to correct for the projection effects so that the space speed of CMEs could be obtained for space weather applications.

\section{ACKNOWLEDGMENTS}

This work was supported by NASA, Air Force Office of Scientific Research, and NSF. 


\section{REFERENCES}

Ambatha, A., M. J., Hagyard, and E. A. West, Evolutionary and Flare-Associated Magnetic Shear Variations Observed in a Complex Flare-Productive Active Region, Solar Phys., 148, 277, 1993.

Antiochos, S. K., Dahlburg, R. B., and Klimchuk, J. A., Astrophys. J., 420, L41, 1994.

Boischot, A., Compt. Rend., 244, 1326, 1957.

Bothmer, V. and Schwenn, R., Adv. Space Res., 70, 215, 1994.

Bougeret, J.-L., et al., Space Sci. Rev., 71, 231, 1995.

Burlaga, L., Sittler, E., Mariani, F., and Schwenn, R., J. Geophys. Res., 86, 6673, 1981.

Cane, H. V., Astron. Astrophys., 1984.

Cane, H. V. et al., Geophys. Res. Lett., 8, 1285, 1981.

Cane, H. V. Sheeley, N. R., Jr., and Howard, R. A., J. Geophys. Res., 92,9869, 1987.

Canfield, R. C., H. S. Hudson, and D. E. McKenzie, Geophys. Res. Lett., 26, 627, 1998.

Chen, J., Geophysical Monograph, 99, 65, 1997.

Chen, J. et al., Astrophys. J., 553, 481, 2000.

Chen, P. F. and Shibata, K., Astrophys. J., 545, 524, 2000.

Cliver, E. W., D. F. Webb, and R. A. Howard, Solar Phys., 187, 89, 1999.

Dodson, H. W., and R. R. McMath., Astrophys. J., 115, 78, 1952.

Falconer, D. J. Geophys. Res., in press, 2001.

Feynman, J. and S. F. Martin, J. Geophys. Res., 100, 3355, 1995.

Filippov, B. P., Astron. Astrophys., 324, 324, 1996.

Filippov, B. P. and O. G. Den, J. Geophys. Res., in press, 2001.

Forbes, T. G., P. A. Isenberg, \& E. R. Priest, Sol. Phys., 150, 245, 1994.

Galvin, A., Geophysical Monograph, 99, 253, 1997.

Glover, A. et al., Geophys. Res. Lett., 27, 2161, 2000.

Gold, T., in Gas Dynamics of Cosmic Clouds, North Holland Publ. C., Amsterdam, p. 103, 1955.

Gopalswamy, N., in Solar Physics with Radio Observations, Eds. T. S. Bastian, N. Gopalswamy and K.

Shibasaki, NRO Report No. 479., p. 141, 1999.

Gopalswamy, N., Geophysical Monograph, 119, 123, 2000.

Gopalswamy, N. and Hanaoka, Y., Astrophys. J., 498, L179, 1998.

Gopalswamy, N. et al., Geophys. Res. Lett., 25, 2485, 1998.

Gopalswamy, N., Hanaoka, Y., and Hudson, H. S., Adv. Space Res., 25 (9), 1851, 2000a.

Gopalswamy, N., et al., Geophys. Res. Lett., 27, 1427, $2000 \mathrm{~b}$.

Gopalswamy, N., Kaiser, M. L., Sato, J. and Pick, M. in High Energy Solar Physics, Ed. R. Ramaty and N.

Mandzhavidze, PASP Conf Ser., vol. 206, p.351 2000c.

Gopalswamy, N. and Kaiser, M. L., Adv. Space Res., in press, 2001.

Gopalswamy, N., and Kundu, M. R., Solar phys., 114, 347, 1987.

Gopalswamy, N., and Kundu, M. R., Solar phys., , , 1989.

Gopalswamy, N. and Kundu, M. R., Astrophys. J., 390, L37, 1992.

Gopalswamy, N., et al., New Astron., 1, 207, 1996.

Gopalswamy, N. et al., Astrophys. J., 486, 1036, 1997.

Gopalswamy, N. et al., Geophys. Res. Lett., 27, 145, 2000d.

Gopalswamy, N., Nitta, N., Manoharan, P. K., Raoult, A. and Pick, M., Astron. Astrophys., 347, 684, $1999 \mathrm{c}$.

Gopalswamy, N. and Thompson, B. J., JASTP, 62, 1457, 2000.

Gosling, J. T., Geophysical. Monograph 58, 343, 1990.

Gosling, J. T., McComas, D. J., Phillips, J. L., Bame, S. J., J. Geophys. Res., 96, 7831, 1991.

Hanaoka, Y. et al., PASJ, 46, 205, 1994.

Hanaoka, Y. and Shinkawa, Astrophys. J., 263, 1998.

Howard, R. A., Michels, D. J., Sheeley, N. R., Jr., and Koomen, M. J., Astrophys. J., 263, L101, 1982.

Hudson, H. S., Solar Physics with Radio Observations, Eds.: T. S. Bastian, N. Gopalswamy and K. Shibasaki, NRO Report No. 479., p. 159, 1999.

Hudson, H. S. and Cliver, E. W., J. Geophys. Res., in press, 2001.

Hudson, H., Lemen, J. R., St. Cyr, O. C. Sterling, A. C., and Webb, D. F., Geophys. Res. Lett., 25, 2481, 1998.

Hudson, H. and D. F. Webb, Geophysical Monograph, 99, 27, 1997.

Hundhausen, A. J., Coronal Expansion and Solar Wind, Springer-Verlag, New York, 1972.

Hundhausen, A. J., in The many faces of the sun : a summary of the results from NASA's Solar Maximum

Mission. Eds. K. T. Strong, J. L. R. Saba, B. M. Haisch, and J. T. Schmelz., New York : Springer, p.143, 1999. 
Jackson, B. V. and Webb, D. F., sowi.conf, 97, 1995.

Jackson, B. V., Sheridan, K. V., Dulk, G. A. and McLean, D. J., Proc. Astron. Soc. Australia, 3, no.5, 383, 1978.

Khan, J. I. and Hudson, H. S., Geophys. Res. Lett., 27, 1083, 2000.

Klassen, A., Aurass, H., Mann, G., and Thompson, B. J., Astron. Astrophys. Suppl., 141, 35, 2000.

Lara, A., Gopalswamy, N. and DeForest, C. E., Geophys. Res. Lett., 27, 27, 1435, 2000.

Linker, J. E., R. Lionello, Z. Mikic, and T. Amari, J. Geophys. Res., in press., 2000.

Low, B. C., J. Geophys. Res., in press., 2000.

Low, B. C. and J. R. Hundhausen, Astrophys. J., 443, 818, 1995.

Maia, D., M. Pick, A. Vourlidas, and R. A. Howard, Development of Coronal Mass Ejections: Radio Shock

Signatures, Astrophys. J., 528, L49, 1999.

Manoharan, P. K., et al., Solar Phys., 156, 377, 1995.

Martin, S. and McAllister, A., Geophysical monograph, 99, 127, 1997.

Marque et al., in press, 2000.

McAllister, A. H., Dryer, M., McIntosh, P., Singer, H., and Weiss, L., J. Geophys. Res., 101, 13497, 1996.

Moore, V. and R. A. Harrison, A characterization of discrete solar wind events detected by interplanetary

scintillation mapping, J. Geophys. Res. 99, 27, 1994.

Morrison, P., Phys. Rev., 95, 641, 1954.

Nitta, N. and Akiyama, S., Astrophys. J., 525, L57, 1999.

Neugebauer, M., and Goldstein, R., Geophysical Monograph, 99, 245, 1997.

Payne-Scott, R., Yabsley, D. E., Bolton, J. G., Nature, 150, 256, 1947.

Reiner, M. J. and M. L. Kaiser, High-frequency type II radio emissions associated with shocks driven by coronal mass ejections, J. Geophys. Res, 104, 16979, 1999.

Reiner, M. J. et al., J. Geophys. Res., in press., 2001.

Richardson, I. G., Using energetic particles to probe the magnetic topology of ejecta, Geophysical Monograph, 99, 189, 1997.

Rust, D. M. and Kumar, A., Astrophys. J., 464, L199, 1996.

Sheeley, N. R., Jr. et al., J. Geophys. Res., 90, 163, 1985.

Sheeley, N. R., Jr., Walters, J. H., Wang, Y.-M., Howard, R. A., J. Geophys. Res., 104, 24739, 1999.

Sheridan, K. V., B. V. Jackson, D. J. McLean, G. A. Dulk, Radio observations of a massive, slow moving ejection of coronal material, Proc. Astron. Soc. Australia, 3, 249, 1978.

St. Cyr, O. C. and A. J. Hundhausen, in Solar Wind Six, Ed. V. J. Pizzo, T. E. Holzer and D. E. Sime, NCAR, Boulder, p. 235, 1988.

St. Cyr, O. C., et al., Properties of coronal mass ejections: SOHO LASCO observations from January 1996 to June 1998 J. Geophys. Res, 105., 18169, 2000.

Sterling, A. C. and H. S. Hudson, Astrophys. J., 491, L55, 1997.

Stewart, R. T., Moving Type IV bursts, in Radio Physics of the Sun, Ed. D. J. McLean and N. R. Labrum, Cambridge, p. 361, 1985.

Subramanian, P. and K. P. Dere, Astrophys. J., in press, 2001.

Thompson, B. J., et al. Solar Phys., 193, 161, 2000.

Tokumaru, M., M. Kojima, K. Fujiki, and A. Yokobe, J. Geophys. Res., 105, 10435, 2000.

Tousey, R., Space Res., 13, 713, 1973.

Tylka, A., J. Geophys. Res., in press., 2001.

van Ballegooijen, A. A. and P. C. H. Martens, Astrophys. J., 361, 283, 1990.

Wang, Y.-M., and N. R. Sheeley, Jr., Astrophys. J., 510, L157, 1999.

Webb, D. F., S. W. Kahler, P. S. McIntosh and J. A. Klimchuk, J. Geophys. Res., 102, 24161, 1997.

Webb, D. F., E. W. Cliver, N. U. Crooker, O. C. St. Cyr, and B. J. Thompson, J. Geophys. Res., 105, 7491, 2000.

Wu, S. T., W. P. Guo, S. P. Plunkett, B. Schmieder and G. Simnett, JASTP, 62, 1489, 2000. 